

\section{SOCIEDAD DE LA INFORMACIÓN, DINÁMICAS DE TRABAJO PARA ENRIQUECER EL CONOCIMIENTO Y ELEMENTOS PARA EL FUTURO DISEÑO DE ESPACIOS EDUCATIVOS}

- sta parte realiza un aporte desde el concepto de learning analytics y las acciones según

- el trabajo con modelos con grandes cantidades de datos, mostrando la tendencia de crecimiento y auge en la actualidad de esta área de estudios en bases de indexación bibliográfica, número de autores y número de eventos académicos realizados sobre este tema. 
Comunicación, redes, aprendizaje y desarrollo institucional y social

\title{
10. Learning analytics: estado actual
}

\author{
Álvaro Francisco Quiroga Cubides
}

\section{Introducción}

L

earning analytics puede ser definido como la medición, la recolección, el análisis y la visualización de datos acerca de los estudiantes y sus contextos, con el propósito de comprender y mejorar el proceso de aprendizaje y el ambiente donde él ocurre (Calvet Liñán y Juan Pérez, 2015). Algunos campos de investigación relacionados con learning analytics son análisis de citas, análisis de redes sociales, modelamiento de usuario, modelamiento cognitivo, tutores, descubrimiento de conocimiento en bases de datos, hipermedia adaptativo, e-learning, estadística, ciencias de la computación, psicología, neurociencia y neurocognición, machine learning, inteligencia artificial, teoría de las organizaciones, learning science, scientomettric y sociología (Siemens, 2013).

Las herramientas, técnicas y aplicaciones usadas en learning analytics están relacionadas con educational data mining. Este último se diferencia del primero en que hace énfasis en el análisis de datos y en que está centrado en el objeto del análisis (Siemens, 2013).

Los análisis en educational data mining incluyen áreas como predicción, clustering, minería de relaciones, extracción de datos para el juicio de los humanos, descubrimiento con modelos, modelamiento del conocimiento del usuario, comportamiento y experiencia, perfilamiento de usuarios, modelamiento de dominios de conocimiento, análisis de tendencias, personalización y adaptación. Mientras que los modelos para hacer learning analytics incluyen recolección de datos, almacenamiento, limpieza, integración, representación y visualización y acción (Siemens, 2013).

La técnica más común para educational data mining es el clustering seguida de la clasificación. Con frecuencia, estos análisis se realizan en plataformas de aprendizaje virtual (Mohamad y Tasir, 2013). Los análisis se pueden agrupar en dos áreas: los relacionados con las actividades académico-administrativas y los referidos a las actividades didácticas.

Álvaro Francisco Quiroga-Cubides. Magīster en Ciencias y Computación. Profesor de la Institución Universitaria Politécnico Grancolombiano. e-mail: aquiroga@poligran.edu.co 
Las actividades académico-administrativas comprenden señales del curso, bitácoras y notas (Clow, 2013). Por su parte, las actividades didácticas incluyen foros, chats, redes de amigos y mensajes (Garćia-Peñalvo y Safont, 2014). Estos análisis se pueden extender a herramientas externas, como las redes sociales (Mohamad y Tasir, 2013). Predecir el rendimiento de estudiante, identificar los estilos de aprendizaje y detectar comportamientos no deseados en los estudiantes (Romero, Ventura, 2010) permite a las instituciones de educación reaccionar con tiempo para lograr el éxito de sus estudiantes.

La educación comienza a vivir en la red como muchas otras cosas. Computational social science es un campo emergente que potencia la capacidad de recoger y analizar datos a escalas que pueden revelar patrones de comportamiento individuales o de grupo (Lazer et al., 2009). Nuestra actividad diaria se puede rastrear a través del uso del móvil, de internet o de los medios de pago electrónicos. En las plataformas de educación en línea, este rastreo es mucho mayor, porque todas las actividades de enseñanza-aprendizaje se realizan a través de aquellas. Este incremento de datos se denomina big data y necesita un modelo para ser manejado: la recolección, la integración, la limpieza, la reducción, la indexación y el análisis (Chen et al., 2013). La diferencia del modelo big data con educational data mining es la etapa de reducción que es necesaria antes de proceder al análisis debido a los volúmenes de información.

En learning analytics, existen varios retos:

- El uso errado de las matemáticas y la tecnología (Clow, 2013): los profesores deben comprender los resultados de learning analytics para entender mejor los procesos de enseñanza-aprendizaje y no convertirse un agente manejado por unos indicadores incomprensibles.

- La apertura de los datos recogidos por las plataformas en la nube (Chorianopoulos, Giannakos, Chrisochoides y Reed, 2014): hoy, muchas instituciones utilizan servicios en la nube con el costo de perder la capacidad de hacer análisis.

- El uso de la información generada en el proceso de enseñanza-aprendizaje: la privacidad y el uso ético de la información es un reto, porque el empleo de la información genera una oportunidad de crear valor comercial (Siemens, 2013).

- Primar la perspectiva de los estudiantes más que la perspectiva de las instituciones (Ferguson, 2012).

- La fertilización cruzada entre la comunidad educational data mining con la comunidad de sistemas inteligentes (Baker e Inventado, 2014). 
Comunicación, redes, aprendizaje y desarrollo institucional y social

\section{Bibliometría}

A continuación, se relacionan diferentes búsquedas, incluyendo sus criterios, y los resultados obtenidos.

Tabla 10.1. Criterios de búsqueda

\begin{tabular}{|l|l|l|}
\hline Fuente & Búsqueda & Documentos \\
\hline Web of Science & TS=“learning analytics” & 226 \\
\hline Scopus & Key (learning analytics) & 760 \\
\hline
\end{tabular}

Fuente: Elaboración propia.

\section{Web of Science}

El número de publicaciones y referencias por año que arroja el buscador se muestra en las figuras 10.1A y 10.1B. Es necesario notar que los datos están tomados a julio de 2015.

Figuras 10.1A y 10.1B. Documentos publicados y número de citas por año en Web of Science.

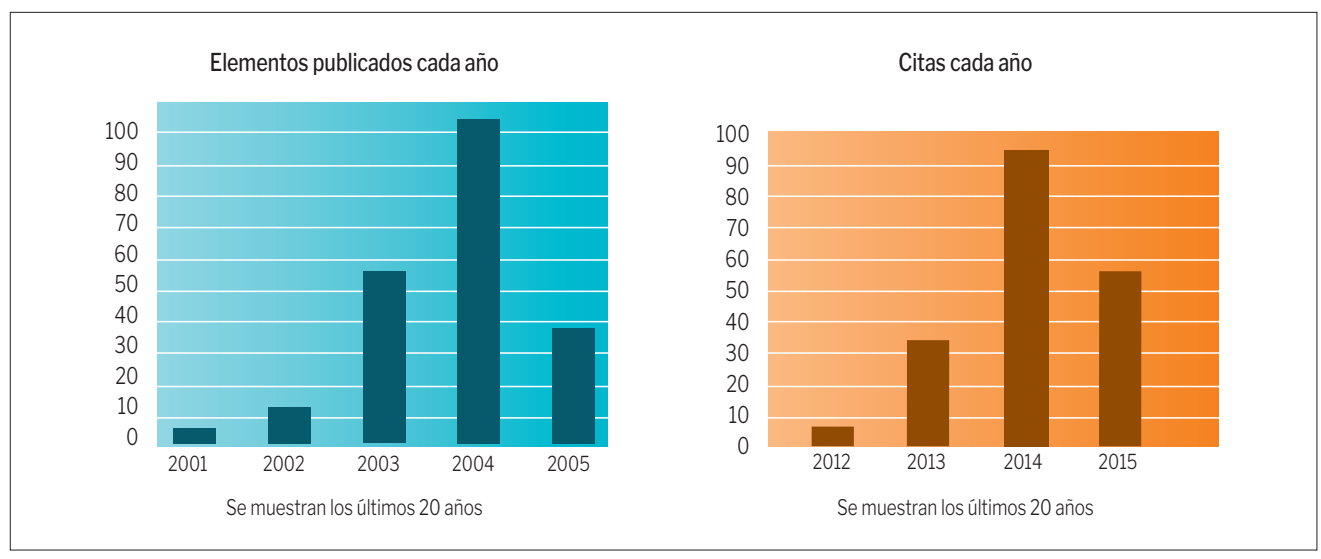

Fuente: Elaboración propia con base en Web of Science.

El indicador $h$-index de Web of Science muestra el valor 7. Esto significa que los 7 documentos más citados tienen al menos 7 referencias. Por su parte, los dominios de investigación que muestra el buscador son science technology, social sciences y arts humanities. Asimismo, las áreas de investigación que muestra el buscador son computer science, educational research, engineering, psychology y social sciences other topics.

Por otro lado, los autores que muestra el buscador son Kloss C. D., Munoz-Merino P. J., Pardo A., García-Peñalvo, F. J. y Theron R. Además, las fuentes más relevantes que muestra el buscador 
son Lecture Notes in Computer Science, IEEE International Conference on Advanced Learning Technologies, 14th IEEE International Conference on Advanced Learning Technologies (ICALT2014), Computers in Human Behavior y IEEE Global Engineering Education Conference.

\section{Scopus}

La figura 10.2 muestra el crecimiento que ha tenido la publicación de documentos en los últimos años. Los datos están tomados a julio de 2015.

Figura 10.2. Número de documentos publicados por año indexados en Scopus.

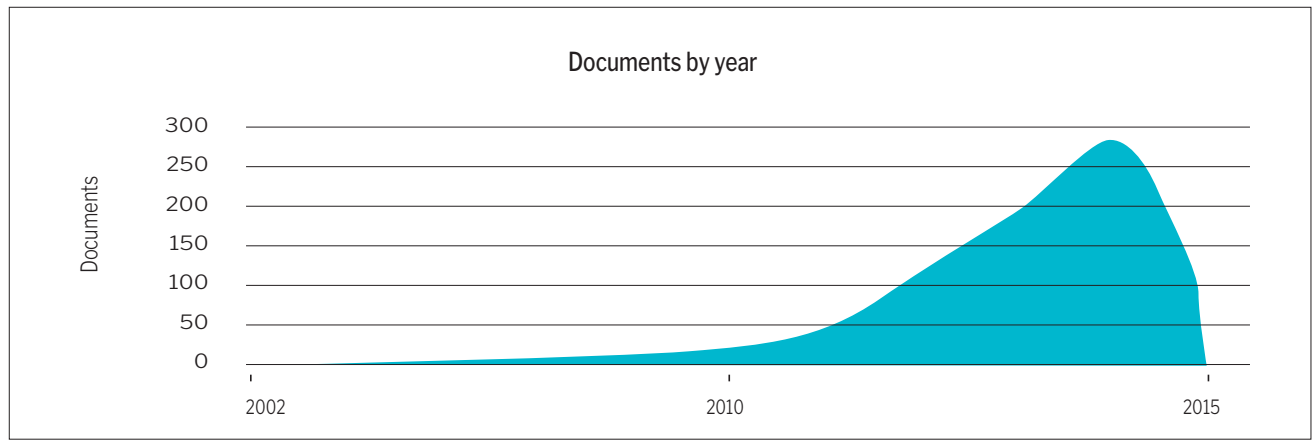

Fuente: Elaboración propia con base en Scopus.

La figura 10.3 enseña que el número de documentos publicados en Lecture Notes en computer science es el mayor. También se ve un aumento importante en el tipo de fuente Computer in Human Behavior.

Figura 10.3. Número de documentos indexados según el tipo de fuente.

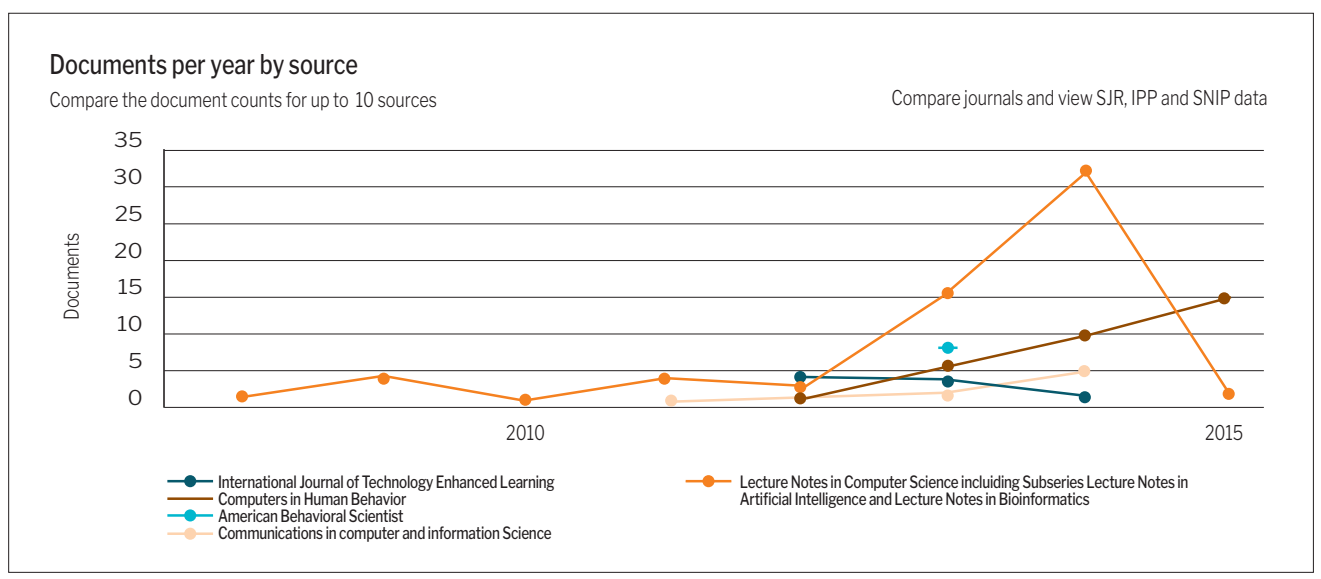

Fuente: Elaboración propia con base en SJR, IPP and SNIP data. 
Comunicación, redes, aprendizaje y desarrollo institucional y social

La figura 10.4 Número de documentos indexados en Scopus por autor muestra que el autor que más publica es Dawson.

Figura 10.4. Número de documentos indexados en Scopus por autor.

Documents by author

Compare the document counts for up to 10 authors

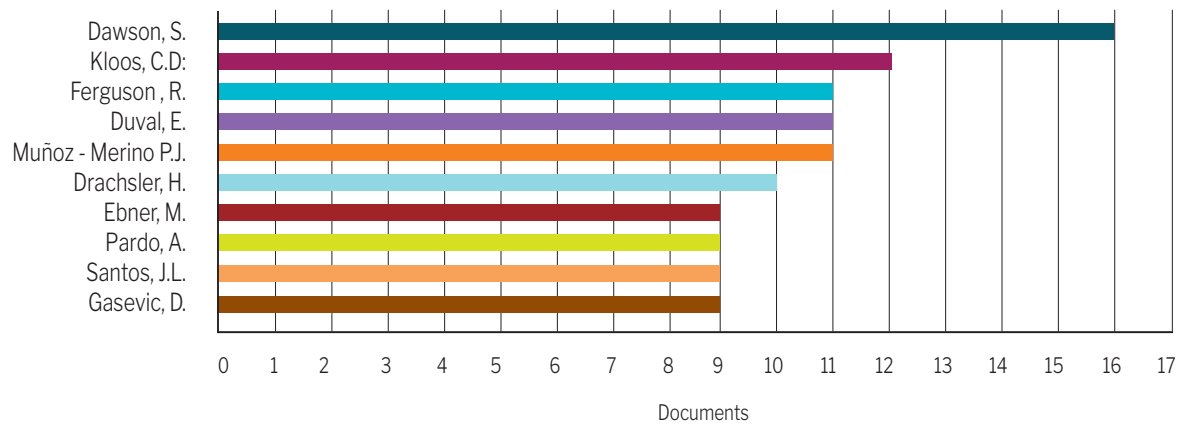

Fuente: Elaboración propia con base en Scopus.

La figura 10.5 expone que el mayor número de publicaciones provienen de autores relacionados con la Open University.

Figura 10.5. Número de documentos indexados en Scopus por institución.

Documents by affiliation

Compare the document counts for up to 15 affiliations

Open University

Universidad Carlos III de Madrid Open University of the Netherlands AthabascaUniversity

Technische Universitat Graz

The University of British Columbia

Rheinisch-Westfallsche Technische Hochschule Aachen

Katholieke Universiteit Leuven Standford University

University Michigan Ann Arbor

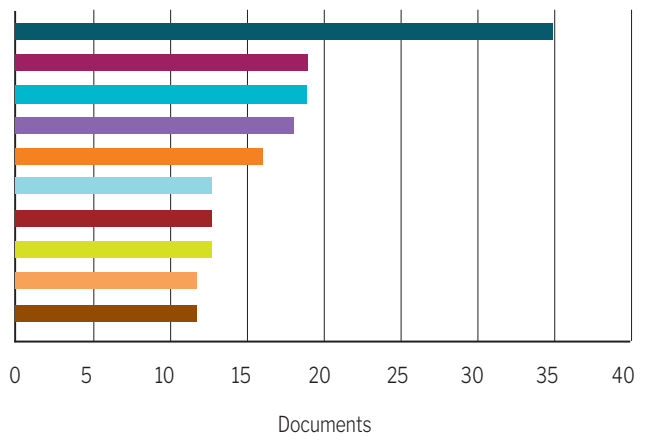

Fuente: Elaboración propia con base en Scopus. 
La figura 10.6 muestra que el país con mayor número de publicaciones es los Estados Unidos.

Figura 10.6. Número de documentos indexados en Scopus por país.

Documents by country/territory

Compare the document counts for up to 15 countries/territories

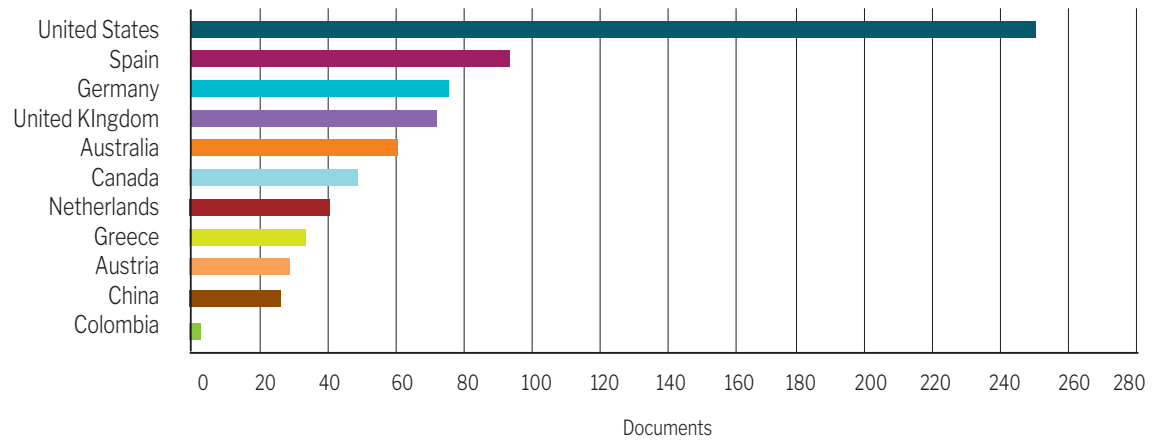

Fuente: Elaboración propia con base en Scopus.

La figura 10.7 indica que el mayor número de publicaciones proviene de conferencias. Esta es una situación típica del área de computer science.

Figura 10.7. Número de documentos indexados en Scopus por tipo de documento.

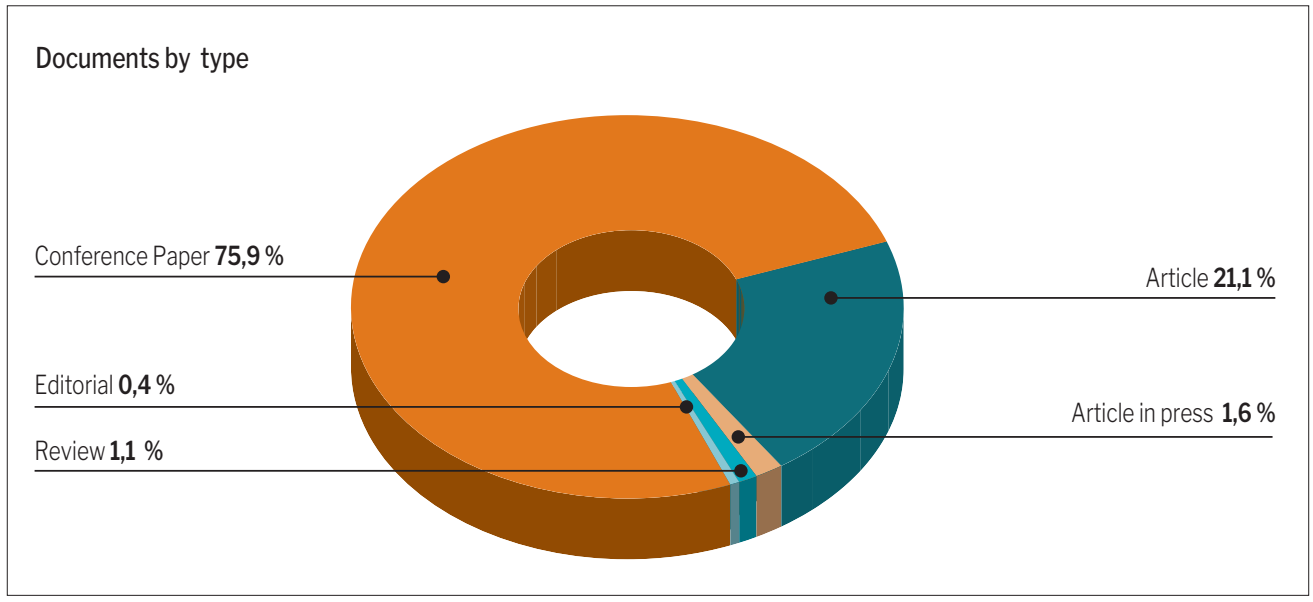

Elaboración propia con base en Scopus. 
Comunicación, redes, aprendizaje y desarrollo institucional y social

La figura 10.8 señala que el área de conocimiento que más publica es computer science.

Figura 10.8. Número de documentos indexados en Scopus por área.

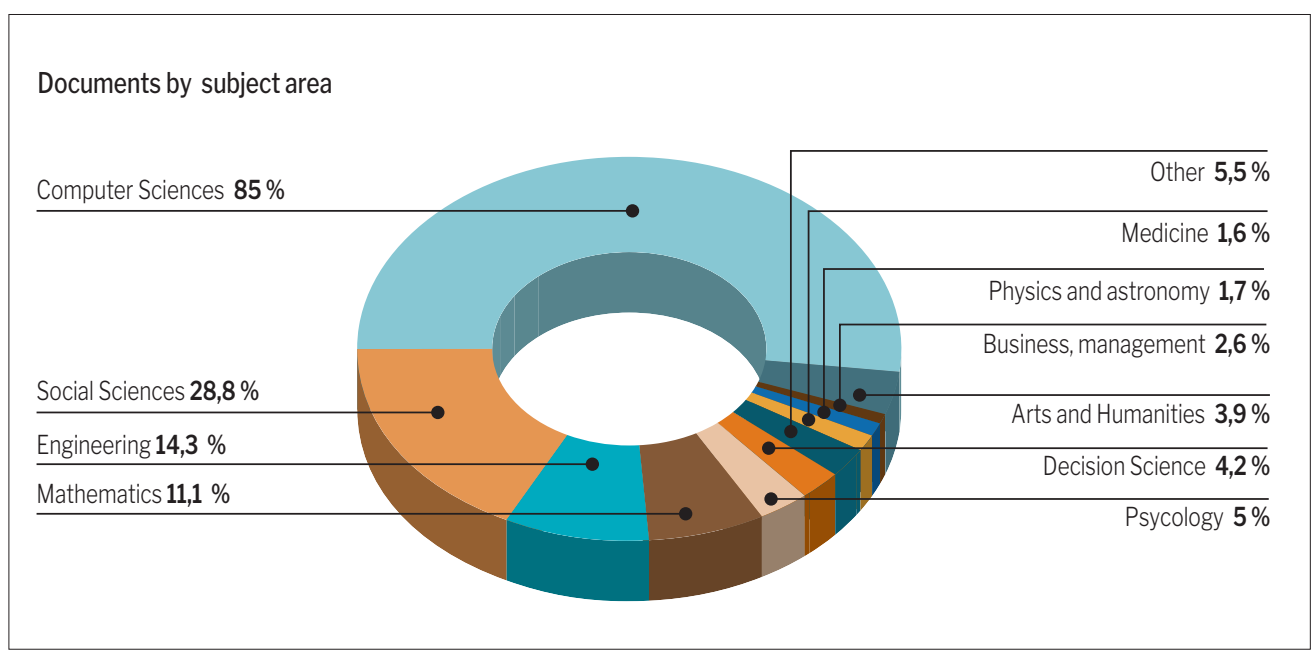

Fuente: Elaboración propia con base en Scopus.

\section{Publicaciones}

\section{Journals}

\section{Lecture Notes in Computer Science ${ }^{1}$}

URL: http://www.springer.com/computer/Incs?SGWID=0-164-0-0-0

Subject Area: Computer Science, Mathematics: Theoretical Computer Science

Publisher: Springer Verlag

ISSN: 0302-9743

SJR (SCImago Journal Rank) (2013): 0.310 (Q2 Computer Science)

IPP (Impact per Publication) (2013): 0.406

SNIP (Source Normalized Impact per Paper) (2013): 0.516

SJR URL: http://www.scimagojr.com/journalsearch.php?q=25674ytip=sid

1 Fuente Scopus. 


\section{Computers in Human Behavior ${ }^{2}$}

Subject Area: Arts and Humanities: Arts and Humanities (miscellaneous), Computer Science: Human-Computer Interaction, Psychology

Publisher: Elsevier Limited

ISSN: 0747-5632

SJR (SCImago Journal Rank) (2013): 1.791

IPP (Impact per Publication) (2013): 3.281

SNIP (Source Normalized Impact per Paper) (2013): 2.406

\section{British Journal of Educational Technology ${ }^{3}$}

Subject Area: Social Sciences: Education

Publisher: Wiley-Blackwell

ISSN: 0007-1013

E-ISSN: $1467-8535$

SJR (SCImago Journal Rank) (2013): 1.523

IPP (Impact per Publication) (2013): 1.930

SNIP (Source Normalized Impact per Paper) (2013): 1.709

\section{Conference Proceeding}

ACM International Conference Proceeding Series:

https://www.acm.org/publications/icp_series

IEEE International Conference on Advanced Learning Technologies:

http://ask4research.info/icalt/2015/

IEEE Global Engineering Education Conference

http://www.educon-conference.org/

2 Fuente Scopus.

3 Fuente Scopus. 


\section{Autores}

\section{Dawson, Shane P.}

University of South Australia, Adelaide, Australia

Documents: 38

Citations: 455 total citations by 376 documents

h-index: 10

Co-authors: 31

Subject area: Computer Science, Social Sciences

\section{Kloos, Carlos Delgado}

Universidad Carlos III de Madrid, Department of Telematic Engineering, Madrid, Spain Documents: 122

Citations: 305 total citations by 283 documents

h-index: 10

Co-authors: 123

Subject area: Computer Science, Engineering

\section{Ferguson, Rebecca I.}

Open University, Institute of Educational Technology, Milton Keynes, United Kingdom Documents: 34

Citations: 114 total citations by 107 documents

h-index: 4

Co-authors: 51

Subject area: Computer Science, Social Sciences

\section{Duval, Erik}

Katholieke Universiteit Leuven, Department of Computer Science, Leuven, Belgium Documents: 124

Citations: 1099 total citations by 845 documents

h-index: 17

Co-authors:150

Subject area: Computer Science, Social Sciences 


\section{Muñoz-Merino, Pedro J.}

Universidad Carlos III de Madrid, Department of Telematic Engineering, Madrid, Spain

Documents: 40

Citations: 122 total citations by 110 documents

h-index: 7

Co-authors: 32

Subject area: Computer Science, Engineering

\section{Pardo, D.}

Ikerbasque, the Basque Foundation for Science, Bilbao, Spain

Documents: 584

Citations: 7349 total citations by 5503 documents

h-index: 44

Co-authors:150

Subject area: Computer Science, Engineering

\section{García Peñalvo, Francisco José}

Universidad de Salamanca, Research Institute for Educational Sciences, Salamanca, Spain

Documents: 127

Citations: 289 total citations by 225 documents

h-index: 9

Co-authors: 140

Subject area: Computer Science, Social Sciences

\section{Therón, Roberto}

Universidad de Salamanca, Department of Computer Science and Automatic, Salamanca, Spain

Documents: 69

Citations: 203 total citations by 165 documents

h-index: 8

Co-authors: 65

Subject area: Computer Science, Mathematics

A continuación, se presentan los autores de nacionalidad colombiana:

\section{Quintero, Camilo Andrés}

Universidad del Valle, Escuela de Ingeniería de Sistemas y Computación, Cali, Colombia

Documents: 1

Citations: 0 total citations by 0 document

h-index: 
Co-authors: 2

Subject area: Computer Science

\section{Florian-Gaviria, Beatriz}

Universidad del Valle, Escuela de Ingeniería de Sistemas y Computación, Cali, Colombia

Documents: 5

Citations: 4 total citations by 4 documents

h-index: 1

Co-authors: 11

Subject area: Computer Science, Engineering

A continuación, se presentan algunos proyectos interesantes relacionados con learning analytics.

\section{http://adlnet.gov/}

ADL (Advanced Distributed Learning) es un proyecto del Gobierno de los Estados Unidos que busca identificar y proveer altos estándares para lograr una educación efectiva y de calidad. En principio, el proyecto surgió para establecer estos estándares en los proveedores de formación del Gobierno, pero luego extendió sus alcances. Trata temas como arquitectura para la enseñanza, juegos, diseño instruccional, tutores inteligentes, educación móvil, educación adaptativa, SCORM, mundos virtuales, registro y recuperación de experiencias de aprendizaje, entre otros.

\section{http://solaresearch.org/}

SoLAR (Society for Learning Analytics Research) es una red interdisciplinaria de investigadores que exploran la función y el impacto de la analítica (learning analytics) en el desarrollo de la enseñanza y el aprendizaje.

\section{http://www.moocresearch.com/}

Es un proyecto apoyado por la Fundación Gates a través de Athabasca University con el fin de explorar el potencial de los MOOC.

\section{http://hedonometer.org/index.html}

Es un servicio de sentiment analysis implementado sobre Twitter basado en el trabajo de investigación de Peter Dods. 
En la figura 10.9, se mencionan las relaciones de algunos conceptos asociados al tema. Se pretende relacionar principalmente dos áreas: data science y learning analytics. Esta relación es muy importante, dado que se plantea que el learning analytics es una aplicación de data science al ámbito de la educación.

Figura 10.9. Mapa conceptual de learning analytics.

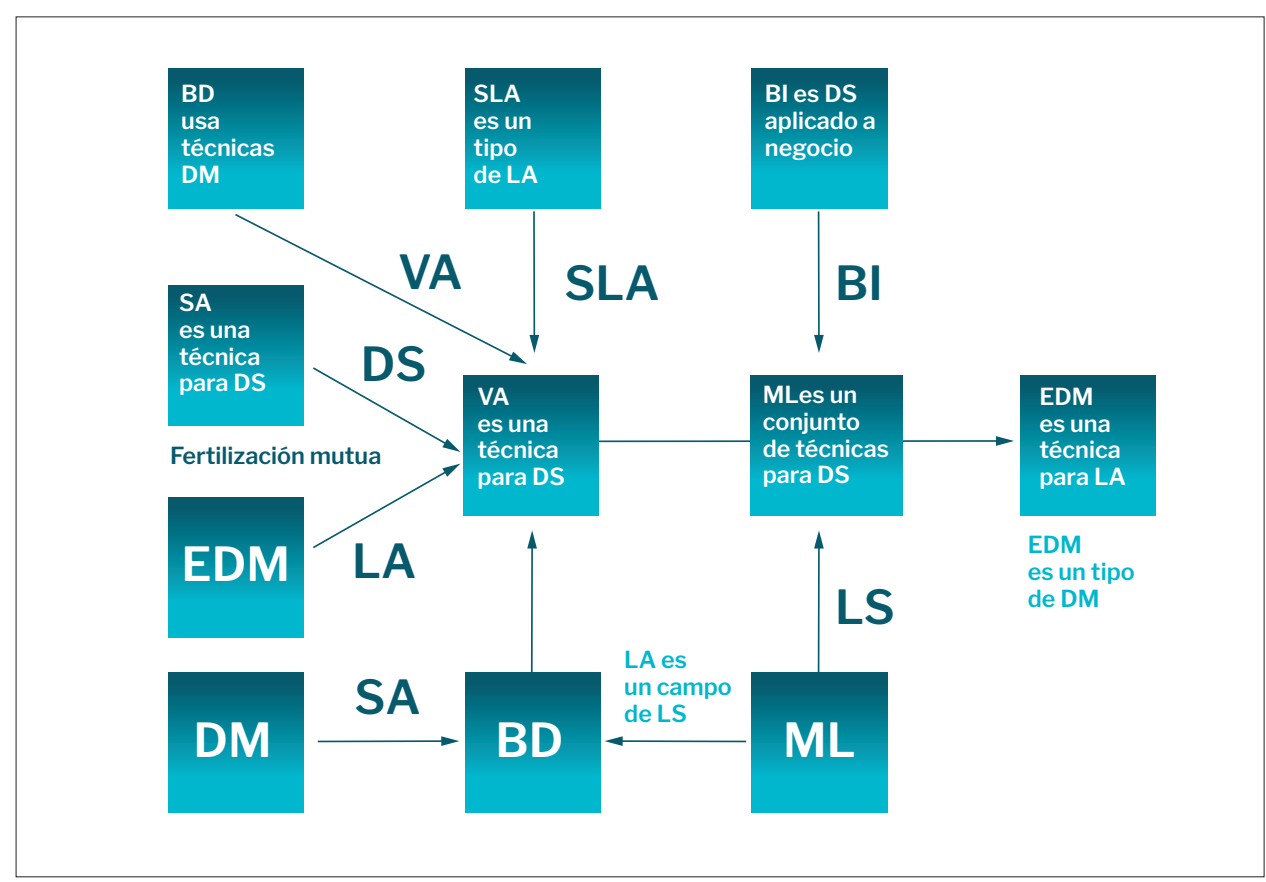

Fuente: Elaboración propia con base en learning analytics.

\section{Learning analytics}

Learning analytics es la medición, recolección, análisis y reporte de datos acerca del proceso de aprendizaje y su contexto, con el propósito de entender y optimizar el proceso y el ambiente donde este ocurre (Siemens, 2013). Learning analytics es un campo de trabajo de learning science. Learning science tiene el objetivo de comprender y mejorar en general los procesos de educación, mientras que learning analytics tiene el mismo objetivo, pero a través del análisis de datos. Learning analytics debe estar en continua relación con data science para mantenerse actualizado con los desarrollos en análisis de datos que alli se logren. 
Comunicación, redes, aprendizaje y desarrollo institucional y social

\section{Educational data mining}

Educational data mining es data mining aplicado a los procesos de enseñanza-aprendizaje con el fin de comprenderlos y mejorarlos (Romero y Ventura, 2010). Asimismo, brinda las técnicas de análisis de datos que son usadas por learning analytics.

\section{Learning science}

Learning science es un campo interdisciplinario que estudia los procesos de enseñanza-aprendizaje con el objetivo de entenderlos y mejorarlos (Sawyer, 2006). Su espacio de trabajo es bastante amplio al ir desde los procesos individuales del estudiante en el momento del aprendizaje hasta los procesos sociales que se llevan en él. Sus técnicas de investigación varían desde técnicas cualitativas a técnicas cuantitativas.

\section{Data science}

Data science es un campo que realiza el estudio sistemático de la organización, las propiedades y el análisis de los datos para realizar procesos de inferencia de conocimiento (Dhar, 2012). Puede ser visto como la unión entre computación, matemática y estadística y un área específica (Conway, 2010). Es este sentido, learning analytics sería la aplicación de data science al área de educación.

\section{Social learning analytics}

Social learning analytics se centra en el estudio del aprendizaje basado en la teoría del aprendizaje y en los elementos de la cultura de la participación en línea (Shum y Ferguson, 2012). En el campo de análisis de datos, el análisis de las redes sociales ha tenido gran interés, el cual no podría faltar en los procesos de aprendizaje dada la naturaleza social de este.

\section{Visual analytics}

Visual analytics es un campo de investigación multidisciplinario que incluye, entre otros, procesos de razonamiento visual y técnicas de visualización de información (Thomas y Cook, 2006). Todo proyecto que incluye data science debe tener el componente de presentación de los resultados de manera óptima para que logre transmitirlos a quien los ve.

\section{Business inteligence}

Business inteligence son técnicas, tecnologías, sistemas, prácticas y aplicaciones que analizan datos críticos de un negocio para ayudar a las compañías a comprender mejor sus negocios y su mercado para tomar las decisiones correctas (Chen, Chiang y Storey, 2012). Se podría decir que es la aplicación de data science al mundo de los negocios. 


\section{Data mining}

Data mining es la acción de explorar datos de manera automática para encontrar conocimiento relevante en ellos (Wu, Zhu, Wu y Ding, 2014). Es el medio que utiliza data science para encontrar información relevante en conjuntos de datos.

\section{Sentiment analysis}

Sentiment analysis es un área de estudio que usa procesamiento de lenguaje natural con el objetivo de identificar el estado de ánimo de las personas de acuerdo con lo que escribe (Bhadane, Dalal y Doshi 2015). Es una de las técnicas para hacer data science. En el caso de learning analytics, es una herramienta que puede ser invaluable en el análisis de las actividades de los estudiantes donde se utiliza texto escrito.

\section{Big data}

Cuando se realizan análisis a grandes volúmenes de datos de diversa índole, se habla de big data. Normalmente, las herramientas de análisis de datos realizan todo el proceso en memoria; pero, cuando el volumen es grande, se deben utilizar técnicas especializadas (Wu et al., 2014). Big data es el uso de data mining sobre grandes volúmenes de información de diversa índole (texto, video, audio, bases de datos, etc.).

Cuando se hace learning analytics, normalmente se realiza sobre información almacenada en las plataformas de gestión de aprendizaje que guardan toda la interacción del estudiante con el ambiente de aprendizaje. El volumen de esta información en plataformas con un gran número de estudiantes puede generar información categorizable como big data.

\section{Machine learning}

Son técnicas que permiten la inducción estadística para generar modelos predictivos (Dhar, 2012). Estas técnicas son usadas en general por data science y en particular por las herramientas de data mining.

\section{Bibliografía anotada}

Se mencionan algunos artículos relevantes para learning analytics.

Siemens, G. (2013). Learning analytics: The emergence of a discipline. American Behavioral Scientist, 57(10), 1380-1400.

Este artículo muestra la definición de learning analytics y sus alcances. 
Gašević, D., Dawson, S. y Siemens, G. (2014). Let's not forget: Learning analytics are about learning. TechTrends, 59(1), 64-71. doi:10.1007/s11528-014-0822-x

Este artículo resalta el objetivo de learning analytics sobre la comprensión y el mejoramiento de los procesos de educación y el riesgo de centrarse en las técnicas olvidando el objetivo.

Gasevic, D., Kovanovic, V., Joksimovic, S. y Siemens, G. (2014). Where is research on massive open online courses headed? A data analysis of the MOOC Research Initiative. The International Review of Research in Open and Distributed Learning, 15(5), 134-176.

Este artículo presenta el resultado de una convocatoria de trabajos de investigación sobre MOOC y cuestiona la falta de investigación, desde la perspectiva de learning analytics, en los actuales procesos de educación masivos (MOOC).

Dawson, S., Gašević, D., Siemens, G. y Joksimovic, S. (2014). Current state and future trends. En Proceedins of the Fourth International Conference on Learning Analytics and Knowledge - LAK'14 (pp. 231-240). Nueva York: ACM Press. doi:10.1145/2567574.2567585

Este artículo muestra los posibles desarrollos del área a la fecha de este.

Lee, P. M., Jheng, S. Y. y Hsiao, T. C. (2014). Towards automatically detecting whether student is in flow. En International Conference on Intelligent Tutoring Systems (pp. 11-18). Springer Verlag. doi:10.1007/978-3-319-07221-0-2

Este es un ejemplo muy interesante de la aplicación de learning analytics en el comportamiento de los estudiantes durante el proceso de aprendizaje. 


\section{Conclusiones y sugerencias}

El learning analytics es un campo que muestra ser de gran potencial para el desarrollo de la educación, sin embargo, es necesario recordar algunos de los retos a los que se enfrenta su aplicación. Uno de ellos es el trabajo conjunto con las áreas especializadas en sistemas inteligentes (Baker, s. f.), de tal forma que el desarrollo desde la perspectiva de métodos y técnicas esté actualizado en forma permanente.

Por otro lado, no se debe olvidar la intención del learning analytics en la comprensión de los procesos de aprendizaje para su mejoramiento. No se puede caer en su uso como si fueran indicadores incomprensibles que guían las acciones de los docentes (Clow, 2013). Finalmente, es indispensable tener siempre presente la ética en el empleo de los datos de los estudiantes de tal forma que en ningún momento se viole sus derechos (Siemens, 2013).

\section{Referencias}

Baker, R. S. e Inventado, P. S. (2014). Educational data mining and learning analytics. En J. Larusson y B. White (eds.), Learning analytics. Nueva York: Springer.

Baker, R. S. J. d. (s. f.). Educational data mining and learning analytics. Recuperado de Recuperado de http://www.columbia.edu/ rsb2162/BakerSiemensHandbook2013.pdf

Bhadane, C., Dalal, H. y Doshi, H. (2015). Sentiment analysis: Measuring opinions. Procedia Computer Science, 45, 808-814. doi:10.1016/j.procs.2015.03.159

Calvet Liñán, L. y Juan Pérez, Á. A. (2015). Educational Data mining and learning analytics: Differences, similarities, and time evolution. International Journal of Educational Technology in Higher Education, 12(3), 98-112. https://doi.org/10.7238/rusc.v12i3.2515

Chen, J., Chen, Y., Du, X., Li, C., Lu, J., Zhao, S. y Zhou, X. (2013). Big data challenge: A data management perspective. Frontiers of Computer Science, 7(2), 157-164.

Chen, H., Chiang, R. H. y Storey, V. C. (2012). Business intelligence and analytics: From big data to big impact. MIS Quarterly, 36(4), 1165-1188. Recuperado de https://goo.gl/ujFhG3

Chorianopoulos, K., Giannakos, M. N., Chrisochoides, N. y Reed, S. (2014). Open service for video learning analytics. En Advanced Learning Technologies (ICALT), 2014 IEEE 14th International Conference on (pp. 28-30). Atenas: IEEE. 10.1109/ICALT.2014.19

Clow, D. (2013). An overview of learning analytics. Teaching in Higher Education, 18(6), 683-695. Recuperado de http://www.tandfonline.com/doi/abs/10.1080/13562517. 2013.827653

Conway, D. (2010). The Data Science Venn Diagram. Recuperado de http://www.dataists. com/2010/09/the-data-science-venn-diagram/ 
Comunicación, redes, aprendizaje y desarrollo institucional y social

Dhar, V. (2012). Data science and prediction. Communications of the ACM, 56(12), 64-73. doi:10.2139/ssrn.2086734

Ferguson, R. (2012). Learning analytics: Drivers, developments and challenges. International Journal of Technology Enhanced Learning, 4(5/6), 304-317. doi:10.1504/IJTEL.2012.051816

Garćia-Peñalvo, F. J. y Safont, L. V. (2014). Human behaviors in computer-based education systems. Computers in Human Behavior, 31(1), 432-433.

Lazer, D., Pentland, A., Adamic, L., Aral, S., Barabasi, A.-L., Brewer, D. ... y Van Alstyne, M. (2009). Social science: Computational social science. Science, 323(5915), 721-723.

Mohamad, S. K. y Tasir, Z. (2013). Educational data mining: A review. Procedia-Social and Behavioral Sciences, 97, 320-324. https://doi.org/10.1016/j.sbspro.2013.10.240

Romero, C. y Ventura, S. (2010). Educational data mining: A review of the state of the art. IEEE Transactions on Systems, Man, and Cybernetics, Part C (Applications and Reviews), 40(6), 601-618. 10.1109/TSMCC.2010.2053532

Sawyer, R. (2006). The new science of learning. En R. K. Sawyer (ed.), The Cambridge handbook of the learning sciences (pp. 1-16). Cambridge University Press.

Shum, S. B. y Ferguson, R. (2012). Social learning analytics. Educational Technology and Society, 15(3), 3-26. Recuperado de http://www.scopus.com/inward/record. url?eid=2-s2.0-84873838796ypartnerlD=tZOtx3y1

Siemens, G. (2013). Learning analytics: The emergence of a discipline. American Behavioral Scientist, 57(10), 1380-1400. Recuperado de http://journals.sagepub.com/doi/ abs/10.1177/0002764213498851

Thomas, J. J. y Cook, K. A. (2006). A visual analytics agenda. IEEE Computer Graphics and Applications, 26(1), 10-13. 10.1109/MCG.2006.5

Wu, X., Zhu, X., Wu, G.-Q. y Ding, W. (2014). Data mining with big data. IEEE Transactions on Knowledge and Data Engineering, 26(1), 97-107. doi:10.1109/TKDE.2013.109 